\title{
Arborescences
}

Revue d'études françaises

\section{Regard rétrospectif sur la norme du français québécois oral}

\section{Davy Bigot}

Numéro 7, décembre 2017

La norme orale en français laurentien

URI : https://id.erudit.org/iderudit/1050966ar

DOI : https://doi.org/10.7202/1050966ar

Aller au sommaire du numéro

\section{Éditeur(s)}

Département d'études françaises, Université de Toronto

ISSN

1925-5357 (numérique)

Découvrir la revue

Citer cet article

Bigot, D. (2017). Regard rétrospectif sur la norme du français québécois oral. Arborescences, (7), 17-32. https://doi.org/10.7202/1050966ar

\section{Résumé de l'article}

Depuis les années 1960, la question de la norme linguistique au Québec a été soulevée à de nombreuses reprises. En 1965, l'Office de la langue française soulignait l'importance d'aligner cette norme sur le français standard international. En 1977, l'Association québécoise des professeurs de français proposait que la norme du français québécois soit basée sur le français formel des Québécois. Cependant, aucune de ces deux propositions n’a réellement fait consensus.

Quelques années plus tard, certains affirmaient qu'il existait une norme québécoise qui n'était basée ni sur du français populaire québécois ni sur du français standard de France, mais que cette norme n'était encore décrite nulle part. Depuis, plusieurs études ont fourni des éléments descriptifs du français standard québécois oral.

Dans notre article, nous nous proposons de dresser un portrait exhaustif du français standard oral en usage au Québec. Dans un premier temps, nous reviendrons brièvement sur le débat entourant la question de la norme linguistique québécoise. Par la suite, nous fournirons une description de cette norme du point de vue phonique et du point de vue grammatical. Enfin, nous terminerons par une réflexion sur l'importance didactique de ce standard québécois dans l'enseignement du français au Québec.
Tous droits réservés ( D Département d'études françaises, Université de Toronto, 2018 document est protégé par la loi sur le droit d’auteur. L’utilisation des services d'Érudit (y compris la reproduction) est assujettie à sa politique d'utilisation que vous pouvez consulter en ligne.

https://apropos.erudit.org/fr/usagers/politique-dutilisation/ 


\section{SOMMAIRE}

1 Marie-Hélène Côté, Université de Lausanne

Anne-José Villeneuve, Université de l'Alberta Introduction

17 Davy Bigot, Université Concordia

Regard rétrospectif sur la norme du français québécois oral

33 Marie-Hélène Côté, Université de Lausanne

Wim Remysen, Université de Sherbrooke

Le "bon usage du français au Québec» selon le Multidictionnaire de la langue française: le cas de la prononciation

49 Anne-José Villeneuve, Université de l'Alberta

Normes objectives et variation socio-stylistique: le français québécois parlé en contexte d'entrevues télévisées

67 Monelle Guertin, Université du Québec à Montréal

Variation sociophonétique dialectale et stylistique: quelle est la langue cible en français langue seconde à Montréal?

90 Suzie Beaulieu, Université Laval

Leif French, Sam Houston State University

Samuel Gagnon, Université Laval

I'veulent-tu parler comme nous-autres? Opinions d'apprenants sur la forme interrogative totale en français laurentien 


\title{
Regard rétrospectif sur la norme du français québécois oral
}

\author{
Davy Bigot, Université Concordia
}

\section{Résumé}

Depuis les années 1960, la question de la norme linguistique au Québec a été soulevée à de nombreuses reprises. En 1965, l'Office de la langue française soulignait l'importance d'aligner cette norme sur le français standard international. En 1977, l'Association québécoise des professeurs de français proposait que la norme du français québécois soit basée sur le français formel des Québécois. Cependant, aucune de ces deux propositions n’a réellement fait consensus. Quelques années plus tard, certains affirmaient qu'il existait une norme québécoise qui n'était basée ni sur du français populaire québécois ni sur du français standard de France, mais que cette norme n'était encore décrite nulle part. Depuis, plusieurs études ont fourni des éléments descriptifs du français standard québécois oral. Dans notre article, nous nous proposons de dresser un portrait exhaustif du français standard oral en usage au Québec. Dans un premier temps, nous reviendrons brièvement sur le débat entourant la question de la norme linguistique québécoise. Par la suite, nous fournirons une description de cette norme du point de vue phonique et du point de vue grammatical. Enfin, nous terminerons par une réflexion sur l'importance didactique de ce standard québécois dans l'enseignement du français au Québec.

\section{Introduction}

En 2005, le Conseil supérieur de la langue française (CSLF) publie Le français au Québec. Les nouveaux défis (Stefanescu et Georgeault 2005), ouvrage divisé en deux parties. La première partie traite du statut de la langue française au Québec, la seconde porte sur sa qualité. Deux ans plus tard, Jean-Claude Corbeil publie chez Québec Amérique L'embarras des langues (Corbeil 2007). Son essai porte sur la politique linguistique au Québec, ainsi que sur la qualité de la langue française des Québécois. Dans ces deux ouvrages, on trouve un nombre non négligeable de recommandations visant à préserver une langue française de qualité. Si la question de la qualité de la langue semble donc être encore d'actualité malgré les nombreux documents académiques, gouvernementaux et autres articles de presse qui ont été rédigés à ce sujet depuis le début $\mathrm{du} \mathrm{xx}^{\mathrm{e}}$ siècle, c'est notamment parce qu'elle est intimement liée à la notion de norme linguistique ${ }^{1}$.

En effet, depuis près de cinq décennies, la question de la norme du français québécois occupe une place de choix dans les discussions. C'est dans le premier cahier rédigé par l'Office de la langue française (OLF 1965) que l'on trouve une définition de cette norme, définition directement basée sur le français dit international. Suite à la Révolution tranquille, l'Association québécoise des professeurs de français (AQPF 1977) en adoptera une nouvelle, cette fois basée sur un français plus québécois ${ }^{2}$.

1. Voir Bigot (2008: 39-44) pour une discussion détaillée.

2. Précisons dès maintenant que par le terme norme, nous entendons ce que Moreau (1997: 219) appelle la norme prescriptive, à savoir «une variété de langue [considérée] comme étant le modèle à rejoindre». 
Depuis, deux camps s'opposent: d'un côté les spécialistes qui font la promotion d'une norme endogène (c'est-à-dire fondée sur l'usage des Québécois), et de l'autre, les partisans d'une norme exogène, pour lesquels la norme du français au Québec doit s'aligner sur le français international, pour ne pas dire hexagonal ${ }^{3}$. Même si le débat n'a toujours pas débouché sur un véritable consensus, il a tout de même généré plusieurs études récentes qui permettent d'éclairer les opinions.

Dans le cadre de cet article, nous nous proposons de dresser un état de la question sur la norme du français québécois, et plus spécifiquement sur la norme orale ${ }^{4}$. Tout d'abord, nous reviendrons sur les différents modèles linguistiques qui ont été adoptés depuis une cinquantaine d'années. Puis nous passerons en revue les éléments descriptifs de cette norme québécoise orale. Enfin, nous terminerons par une discussion dans laquelle nous formulerons des pistes de réflexion visant à faire avancer le débat sur la norme orale du français en usage au Québec.

\section{La question de la norme du français québécois}

Revenons tout d'abord sur le débat de la norme du français québécois et plus précisément sur les différents modèles qui ont été proposés jusqu’à présent.

\subsection{Le modèle international}

En 1965, l'OLF publie son premier cahier consacré à la norme du français écrit et parlé au Québec. La position de l'organisme est, dès le départ, très claire: «l'usage doit s'aligner sur le français international, tout en faisant place à l'expression des réalités spécifiques nord-américaines. (OLF 1965: 6). L'OLF profite de ce premier numéro pour donner une définition de sa norme du français québécois:

Ainsi, la norme qui, au Québec, doit régir le français dans l'administration, l'enseignement, les tribunaux, le culte et la presse, doit, pour l'essentiel, coïncider à peu près entièrement avec celle qui prévaut à Paris, Genève, Bruxelles, Dakar et dans toutes les grandes villes d'expression française.

(OLF 1965: 6)

À cette époque, cette définition n'est pas sans poser problème. En effet, lorsqu'elle est proposée, le Québec est en pleine Révolution tranquille et vit une crise identitaire importante. Les Québécois sont en quête de reconnaissance de leur parler, et leur imposer une norme basée sur un modèle international sera voué à l'échec. Pour autant, si la définition de l'OLF n'a jamais été adoptée, il faudra attendre plus d'une décennie avant qu'une nouvelle définition basée sur une variété plus locale ne soit proposée, cette fois, par l'AQPF. Notons que depuis la publication de son premier cahier, l'OLF s'est abstenu de se prononcer sur la question de la norme du français québécois et a «restreint son champ d'intervention aux terminologies techniques utilisées dans la "communication institutionnelle ou officielle" " (Maurais 1987: 397).

3. À ce sujet, Corbeil (2007: 306) note: «Il n’y aurait [pour les puristes] qu'une langue française et qu' une seule norme, celle de Paris, dont on atténue le caractère centralisateur sous l'euphémisme de français international.»

4. Par norme orale, nous entendons ce qui concerne uniquement la prononciation ainsi que la grammaire. Nous évacuons ce qui touche le lexique pour deux raisons: 1) la question des dictionnaires fait depuis longtemps l'objet d'un débat qu'il ne serait pas possible de résumer dans le présent article (voir notamment Bigot 2008: 149-154) et 2) la question du lexique concerne les registres de langue, mais surtout l'acceptation ou non des mots considérés comme typiquement québécois. Pour le moment, aucun dictionnaire ne semble d’ailleurs faire consensus. 
De nos jours, le modèle international reste encore régulièrement soutenu. Nemni (1998: 167) affirme, par exemple, que «les Québécois francophones utilisent le français international pour le registre formel». Plus récemment, le président du Conseil supérieur de la langue française déclarait que «le seul français qui peut être enseigné est un français national, de niveau international, qui peut être défini différemment, selon qu'il s'agit de l'oral ou de l'écrit. Le français d'ici, de registre "officiel", correspond à ce français international» (CSLF 2007: 2). Cette proposition soulève, selon nous, plus de questions qu'elle n'en résout. Qu'entend-on par "français national de niveau international »? Que signifie registre "officiel»? Quels sont les locuteurs susceptibles d'employer ce français officiel? Enfin, comment peut-on affirmer que ce français d'ici de registre officiel puisse correspondre au français international, alors que l'existence même du français international ne fait même pas consensus chez les linguistes 5 ? À notre connaissance, le CSLF ne s'est, depuis, plus prononcé sur la question de la norme. L'ambiguïté de sa position subsiste encore et ne permet donc en aucun cas de faire avancer le débat de la norme du français québécois.

\subsection{Le modèle québécois}

Dans les années 1970, la question de la norme à enseigner dans les écoles divise les enseignants (Comité interministériel sur la situation de la langue française 1996: 183). En 1977, l'AQPF tient son congrès annuel durant lequel elle tente de répondre à deux questions principales: quel est le français standard d'ici? Et quel français doit-on enseigner au Québec? Ses membres arrivent à la conclusion suivante: «Le français standard d'ici est la variété de français socialement valorisée que la majorité des Québécois francophones tendent à utiliser dans les situations de communication formelle.»(AQPF 1977: 11) ${ }^{6}$. La proposition sera adoptée et le Conseil de la langue française (CLF) la reprendra mot pour mot treize ans plus tard (CLF 1990: 31).

Tout comme la définition de l'OLF, celle de l'AQPF pose un problème majeur: elle ne précise nullement ce que signifie "variété de français socialement valorisée ${ }^{7}$ ». Dans un article publié deux ans après la proposition de l'AQPF, Forget (1979: 156-157) souligne que:

Dans les organismes garants de la culture tels la radio, la télévision et dans l'enseignement, un français québécois avec ses particularités phonologiques, lexicales et morphosyntaxiques est le plus souvent utilisé par les locuteurs. Cette situation qui n'est pas officialisée prévaut néanmoins et a tendance à être de plus en plus admise; elle reçoit l'approbation commune. D'ailleurs, l'élite traditionnelle québécoise des prêtres, médecins, avocats, notaires n’a jamais, il me semble, été visée par les demandes répétées d'épuration de la langue malgré l'aspect dialectal, régional évident de son parler. Il en va de même des professeurs. Le français standard québécois pourrait très bien être accepté et devenir un point de référence culturel.

5. Pour Santerre $(1981,1990)$, ce français international existe bel et bien, alors que selon Verrault (1999), ce français relève du mythe plutôt que d'une réalité linguistique. Voir Bigot (2008: 125-128) pour une discussion plus approfondie à ce sujet.

6. L'Association québécoise des professeurs de français a été créée à Montréal en 1967, avec notamment pour but celui de promouvoir la langue française et la culture québécoise. http://www.aqpf.qc.ca (consulté le 14 août 2015).

7. Cajolet-Laganière et Martel (1995: 13) notent que l'AQPF «a approuvé et reconnu officiellement le modèle articulatoire des annonceurs de Radio-Canada». Nous n'avons pas retrouvé de trace de cette approbation officielle. Nous ne savons donc pas si elle fut antérieure ou non à la définition que l'AQPF a proposée en 1977, et nous ne pouvons, ainsi, pas savoir si oui ou non, la norme radio-canadienne représentait déjà, à cette époque, pour l'AQPF, le modèle à rejoindre, cette "variété de français socialement valorisée ". 
Il existe donc, selon elle, une norme québécoise socialement valorisée (notamment par les élites du Québec) et véhiculée à l'école et dans certains médias culturels, et c'est cette variété de français qui devrait faire figure de français québécois de référence.

Cette position est toutefois loin de faire consensus. Selon Barbaud (1998: 107), le parler des élites québécoises diverge trop du français international pour qu'il puisse servir de modèle normatif. Enfin, pour Lamonde (2004: 62), «l'élite n'est plus la gardienne d'une certaine norme française, mais ni plus ni moins que l'empêcheuse de patoiser et de s'angliciser en rond $^{8}{ }^{\prime}$.

Autrement dit, les avis sont nettement partagés et il semble difficile de définir ce que l'AQPF (1977) entend par "variété de français socialement valorisée». Il faudra attendre plus de vingt ans pour qu'un sondage portant sur la qualité de la langue dans les médias québécois (Bouchard et Maurais 1999) soit réalisé et révèle que le modèle linguistique normatif valorisé par la population québécois est le français des lecteurs de nouvelles de Radio-Canada.

\subsection{Le modèle radio-canadien}

Depuis sa création, Radio-Canada est reconnue pour la qualité de sa langue, et cela plus particulièrement dans ses bulletins de nouvelles. Ce n'est d'ailleurs pas un hasard. Dubuc (2001: 127) précise en effet que:

Par concours de circonstances et aussi par un souci de qualité qui l'honore, Radio-Canada a longtemps fait figure de modèle de langue au Canada français. Il faut remonter aux origines de l'entreprise d'État pour comprendre mieux le phénomène. Dès sa fondation en 1936, ses premiers dirigeants francophones, notamment Augustin Frigon et Marcel Ouimet, ont fait le choix linguistique qui s'est imposé comme la langue des ondes. Le modèle choisi correspondait à la langue de l'élite cultivée du Canada français d'alors.

Le choix du modèle de référence s'est donc, dès le départ, tourné vers une variété socialement prestigieuse, le français de l'élite québécoise cultivée, et une véritable politique linguistique radio-canadienne axée autour de ce parler s'est rapidement mise en place?.

Le 12 septembre 1960, un comité linguistique est officiellement mis sur pied au sein de la Société Radio-Canada (SRC). Les mandats suivants lui sont confiés : 1) améliorer la langue à l'antenne, 2) améliorer la langue de la gestion interne, 3) servir d'organisme de gestion de la langue, et 4) recueillir les matériaux nécessaires à la compilation de la terminologie de la production (radio et télévision) et de la gestion (Dubuc 1990: 136). Ce comité suscitera, par le biais de ses publications (dont les reconnus Que dire? et C'est-à-dire), un grand enthousiasme qui sera couronné en 1963 par la coupe Émile de Girardin, décernée par l'Office du vocabulaire français de Paris, pour sa contribution exceptionnelle à l'amélioration de la qualité du français en radiotélévision. Le Comité linguistique de Radio-Canada ne

8. Notons également la position de l'auteur et essayiste Georges Dor qui déplorait que le parler des journalistes et animateurs de la télévision «[illustrait] avec une éloquence parfois pathétique, l'urgent besoin de nous enseigner, à l'école primaire, à parler à peu près correctement et intelligemment notre langue» (Dor 1998: 10).

9. Gendron (2014: 4) souligne cependant que l'influence de l'élite québécoise est restée pendant longtemps limitée. D'une part, ces membres occupaient un rang social inférieur à celui des anglophones. D'autre part, ils entretenaient des liens privilégiés avec l'administration anglaise, ce qui était souvent mal perçu par le reste de la population francophone. 
sera donc plus un simple outil de standardisation linguistique au sein d'une entreprise (Dubuc 1990: 137).

Naturellement, le français diffusé sur les ondes de la SRC va petit à petit s'imposer comme norme au sein de la population québécoise ${ }^{10}$. Ce statut sera confirmé trente ans plus tard par une étude réalisée auprès de 1591 répondants dans laquelle Bouchard et Maurais (1999) dégagent trois grands modèles normatifs du français parlé au Québec:

Tableau 1: Modèles normatifs constatés et pourcentages de francophones (extrait de Bouchard et Maurais 1999: 100)

\begin{tabular}{|l|c|c|c|c||}
\hline & $\begin{array}{c}\text { Variétés } \\
\text { géographiques }\end{array}$ & $\begin{array}{c}\text { Parler } \\
\text { auto évalué }\end{array}$ & Modèle de référence & $\begin{array}{c}\text { Pourcentage de } \\
\text { francophones }\end{array}$ \\
\hline Modèle 1 & Québécois & $\begin{array}{c}\text { Plutôt à la manière } \\
\text { québécoise }\end{array}$ & $\begin{array}{c}\text { Lecteurs de nouvelles } \\
\text { de Radio-Canada }\end{array}$ & $54 \%$ \\
\hline Modèle 2 & Québécois & $\begin{array}{c}\text { Tout à fait à la } \\
\text { manière québécoise }\end{array}$ & $\begin{array}{c}\text { Gens ordinaires des } \\
\text { jeux télévisés }\end{array}$ & $28 \%$ \\
\hline Modèle 3 & Français & À la manière française & $\begin{array}{c}\text { Lecteurs de nouvelles } \\
\text { de Radio-Canada }\end{array}$ & $17 \%$ \\
\hline
\end{tabular}

Arrêtons-nous un instant sur la composition du tableau. Les deux premières colonnes représentent respectivement le type de modèle normatif favorisé par les répondants et la variété géographique de ce modèle. La troisième colonne considère le parler auto-évalué des répondants (ces derniers considèrent qu'ils parlent à la manière québécoise ou française). La quatrième colonne représente le modèle de référence privilégié par les répondants, c'est-à-dire, le modèle qui, selon eux, présente le meilleur parler. Enfin, la dernière colonne donne les pourcentages de répondants en faveur de chacun des trois modèles normatifs.

Les résultats de Bouchard et Maurais (1999: 100) sont sans appel. En totalisant les pourcentages des modèles un et trois, on peut en conclure que la variété de référence (donc socialement valorisée) du français parlé au Québec est, pour la grande majorité des Québécois, celui des lecteurs de nouvelles de Radio-Canada ${ }^{11}$.

Nous avons vu à la section précédente que le principal problème de la définition de la norme du français québécois proposée par l'AQPF (1977) était l'absence de consensus autour du modèle «socialement valorisé» par la majorité des Québécois. Si ce consensus n’existe pas chez les spécialistes, il semble exister au sein de la population pour qui la norme est le français des lecteurs de nouvelles de Radio-Canada.

Pour autant, le problème est-il résolu? On peut se demander s'il est raisonnable que la norme du français québécois soit le français de certains journalistes. En effet, les lecteurs de nouvelles de RadioCanada sont des locuteurs spécialisés, qui reçoivent une formation linguistique soutenue et régulière

10. Gendron (1990: 374) note déjà plusieurs années avant l'étude de Bouchard et Maurais (1999): «[qu'il] est connu que le modèle radio-canadien symbolise depuis longtemps ce que les Québécois valorisent sur le plan de la prononciation".

11. Notons cependant que pour $17 \%$ des répondants, ce modèle semble davantage représentatif du français de France que du français québécois. Léard (1995) partage d'ailleurs ce point de vue et va même plus loin en affirmant que le français radio-canadien n'est tout simplement pas la langue des Québécois. Ceci n'est d'ailleurs peut-être pas un hasard. Bigot et Papen (2013) soulignent, en effet, que le dictionnaire de prononciation de Warnant (1987), ouvrage de référence à Radio-Canada (Dubuc 1990: 145), est un dictionnaire axé sur la norme parisienne. 
(Bertrand 2005: 452). De plus, ces locuteurs spécialisés sont des lecteurs de nouvelles parce qu'ils lisent les nouvelles, le plus souvent à partir d'un télésouffleur ${ }^{12}$. En cela, leur discours est naturellement beaucoup plus proche de l'écrit que de l'oral. Leur français ne peut donc pas être prescrit à titre de modèle de référence unique du français québécois oral, car ce serait installer une norme impossible à rejoindre pour la quasi totalité de la population. Or, nous pensons que la norme doit refléter en partie les usages réels des Québécois. Un modèle basé uniquement sur le parler de certains journalistes de $\mathrm{SRC}$ resterait donc, selon nous, purement hypothétique, voire, à toutes fins pratiques, inutile.

\subsection{Qui sont les détenteurs de la norme du français québécois?}

Bigot (2008: 16-26) souligne que, depuis plusieurs siècles, la norme linguistique du français est définie à partir des usages des classes sociales dominantes et propose qu'il en soit ainsi au Québec. Cette position ne fait toutefois pas l'unanimité. Maurais (1986) remet en cause le fait que la classe dominante de la population québécoise puisse être détentrice de la norme:

Qui détermine les critères qui servent à évaluer la qualité des discours? On pourrait répondre rapidement en disant que c'est le groupe social dominant, économiquement ou culturellement. Mais, pour ma part, je préferre parler [...] de «couches linguistiquement hégémoniques», c'est-à-dire «les couches dont l'activité langagière est prise comme modèle, est considérée comme centre de gravité de la langue et qui sont hégémoniques du point de vue du pouvoir» [...], même si, dans certaines sociétés, elles peuvent se confondre ou s’intégrer à la classe dominante. (Maurais 1986: 79)

Il ajoute un peu plus loin que

[...] la partie la plus active et surtout la plus visible actuellement de ces couches linguistiquement hégémoniques est constituée par des normalisateurs [...], ceux de l'Office de la langue française, ceux des différentes commissions de terminologie et ceux qui sont chargés de la correction linguistique dans les divers ministères. (Maurais 1986: 79)

Lamonde (2004: 63) abonde dans le même sens que Maurais (1986) en précisant qu'elle n’est "pas la seule à penser qu'on apprendrait des choses fort instructives sur le standard d'ici si on s'intéressait de plus près au français de ce groupe sélect ${ }^{13}$ ».

Auger (1988: 63) propose que la variété de référence soit celle parlée par la couche moyenne supérieure, fortement scolarisée, de la population québécoise. Nous pensons que la définition d’Auger est, elle aussi, trop restrictive, car elle ne prend en compte qu'un sous-groupe très limité de la population québécoise. En d'autres termes, le parler des membres moins scolarisés des couches supérieures québécoises est-il nécessairement différent de celui des membres plus scolarisés? Ce n’est pas évident. Corbeil (1983) explique d'ailleurs qu'il existe

12. Précisons que ce n'est cependant plus la seule forme de présentation de l'information. En effet, Martel et al. (2010) ont démontré notamment que les interactions entre journalistes et chefs d'antenne étaient de plus en plus fréquentes (même à Radio-Canada) et que cela n'était pas sans conséquence sur l'usage du français en ondes.

13. Nous partageons ce point de vue. Néanmoins, nous pensons que restreindre la norme linguistique aux seuls usages de langagiers nous paraît délicat. En effet, comme nous l'avons déjà souligné, ne risque-t-on pas de proposer une nouvelle fois, une norme trop peu représentative des usages réels de la population, dans laquelle la majorité des Québécois ne se reconnaitra pas et qui sera donc possiblement rejetée par la majorité d'entre eux? 
[un] phénomène par lequel les comportements linguistiques de chaque membre d'un groupe ou d'un infragroupe donné sont façonnés dans le respect d'une certaine manière de faire sous l'influence de forces sociales émanant du groupe ou de ses infragroupes. (Corbeil 1983: 283)

Selon ce Principe de régulation linguistique (Corbeil 1983), il est donc tout à fait possible qu'un professeur (qui possède un niveau d'études universitaires élevé) et un chef d'entreprise (dont le niveau scolaire peut ne pas dépasser le niveau secondaire) puissent avoir le même parler, du fait de l'influence des forces sociales qui fait en sorte que des membres d'un groupe social (comme celui de l'élite québécoise) vont tendre vers une même norme linguistique en dépit de leur niveau de scolarisation.

De nombreux travaux en sociolinguistique - citons entre autres Labov (1976) et Trudgill (1995) - ont montré que dans les communautés socialement stratifiées, la norme se définissait à partir des usages des classes sociales élevées (les intellectuels, les écrivains, les artistes, les professionnels des médias, les politiciens, etc.). Pourquoi serait-ce différent au Québec? Corbeil (1986) propose que la norme définie comme modèle à suivre s'illustre à l'oral dans le discours de «locuteurs prestigieux", et qu'elle soit, à l'écrit, la langue de la littérature et des journaux. La question est maintenant la suivante: qui sont ces locuteurs prestigieux?

Pour Dumas (2006), la norme du français québécois devrait être basée sur le français que l'on peut

observer dans les manifestations officielles de la parole publique, essentiellement dans les émissions d'information et d'affaires publiques des réseaux publics (non commerciaux) de radio et de télévision, qui représentent les principales tribunes modernes des instances officielles définissant la norme. (Dumas 2006: 100)

Cette proposition nous semble elle aussi problématique. En effet, que doit-on comprendre par «manifestations officielles de la parole publique »? Une fois de plus, quels sont les locuteurs ciblés par Dumas (2006) ? Désigne-t-il des journalistes en particulier ou l'ensemble des personnes intervenant sur les réseaux publics, à savoir les journalistes et les personnes interviewées? Peu importe la définition proposée, celle-ci parait, pour le moment, soit trop restrictive, soit pas assez précise. Nous proposerons, dans la discussion, une définition susceptible de résoudre ce problème.

\section{Les éléments descriptifs}

Passons maintenant aux études descriptives qui ont traité de la norme du français québécois oral. Tout d'abord, nous nous attarderons sur la prononciation. Puis nous nous pencherons sur la grammaire. Précisons à nouveau que l'objectif est de résumer l'ensemble des traits linguistiques considérés comme étant potentiellement caractéristiques de la norme du français parlé au Québec.

\subsection{La prononciation}

Deux études sociolinguistiques portant sur la prononciation des lecteurs de nouvelles de la SRC ont été effectuées: Cox (1998) et Reinke (2005). À ces recherches, il faut également ajouter l'article de Bigot et Papen (2013) consacré à la question de la norme du français au Québec et plus généralement au Canada. 
Dans son enquête, Cox (1998) propose qu'un modèle de norme du français canadien soit basé sur l'usage canadien. Afin de décrire cette norme, il a étudié un corpus d'enregistrements de "Canadiennes et de Canadiens francophones ayant comme formation de parler/lire devant une caméra" (Cox 1998: 177). Selon lui, ces lecteurs de nouvelles de Radio-Canada (six femmes et cinq hommes originaires des principales villes du Canada), ainsi que le présentateur national de RDI, constituent un échantillon de l'élite qui doit être prise pour modèle de prononciation (Cox 1998: 192, note 8, ne justifie cependant pas sa position). Cox (1998) a observé quatre éléments :

- L'assibilation de /t/ et de /d/ devant /i/ /y/ et les glissantes /j/ et / $\mathrm{u} /$ comme dans tu dis $\left.\left[\mathrm{t}^{\mathrm{s}} \mathrm{yd}^{\mathrm{z}} \mathrm{i}\right]\right)$;

- Le relâchement des voyelles /i, y, u/ comme dans rite [RIt], rut [RYt] et route [Rut];

- Le dévoisement des voyelles /i, y, u/ comme dans équipé [ekipe], député [depyte] et écouté [ekute] ;

- Le «a» postérieur [a] comme dans Canada [kanada], tard [taR] et pâte [pat].

Plus récemment, Reinke (2005) a effectué une analyse de trente-deux variables à partir d'un corpus de 38 émissions télévisuelles diffusées sur Radio-Canada, TVA, TQS et Télé-Québec entre 1999 et 2001. Les variables qu'elle a prises en compte incluent ${ }^{14}$ :

- Le (a\#) comme dans Canada ([a] vs [a] vs [o]);

- Le $(\varepsilon: C \#)$ comme dans neige ([ع:] vs $\left[\mathrm{a}^{\varepsilon}\right]$ vs $\left[\mathrm{a}^{\mathrm{e}}\right]$ vs $\left.\left[\mathrm{a}^{\mathrm{j}}\right]\right)$;

- Le (o:C\#) comme dans côte ([0:] vs $\left[\mathrm{o}^{\mathrm{u}}\right]$ vs $\left.\left[\mathrm{\jmath}^{\circ}\right]\right)$;

- Le (ø:C\#) comme dans meute ([ø:] vs [øу] $)$.

Son corpus est composé de 152 locuteurs. Les 38 émissions sont réparties selon trois types de programmes (Reinke 2005: 16-17) : 1) type 1 : les émissions d'informations, les bulletins de nouvelles, etc., 2) type 2: les entretiens, les jeux télévisés, etc. et 3) type 3: les émissions ludiques, les émissions de variétés, etc.

Les études de Cox (1998) et Reinke (2005) diffèrent sur bien des points et nous renvoyons le lecteur à Bigot et Papen (2013) pour de plus amples détails sur ce qui distingue les deux analyses. Toutefois, plusieurs points de convergence existent entre les deux études, notamment en ce qui a trait aux résultats obtenus. L'article de Bigot et Papen (2013) fait une synthèse des éléments de prononciation qui, à la lumière des résultats de Cox (1998) et Reinke (2005), semblent faire partie de la norme du français québécois. On y dégage, en effet, huit traits saillants de la prononciation des lecteurs de nouvelles (Bigot et Papen 2013: 125):

- L'assibilation de /t/ et /d/ devant /i//y/ et les glissantes / $\mathrm{j} /$ et / $\mathrm{y} /$ comme dans $t$ tu dis ([tydi] vs $\left.\left[\mathrm{t}^{\mathrm{s}} \mathrm{yd}^{\mathrm{z}} \mathrm{i}\right]\right)$;

- La réalisation "complète» des groupes consonantiques finals comme dans table ([tabl] vs $[\mathrm{tab}])$;

- La distinction entre /a/ et /a/ comme dans patte [pat] vs pâte [pat]);

- La non-réalisation de -oi en /we/, /we/ ou /wo/ comme dans bois ([bwe], [bwe] et [bwo]);

14. Voir Reinke (2005: 21-22) pour la liste complète des variables. 
- La non-diphtongaison des voyelles longues comme dans neige ([ne:3] vs [neinj]), à l'exception de /ø:/ et /o:/;

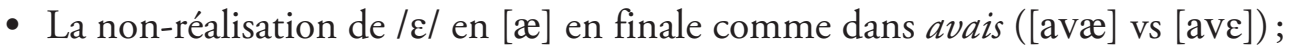

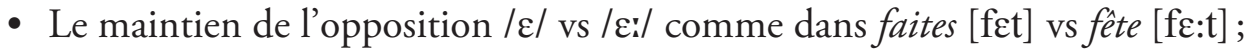

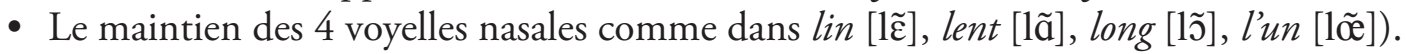

Bigot et Papen (2013: 126) remarquent qu'il ne reste cependant pas moins de six traits dont le statut n’est pas encore fixé:

- L'effacement de /1/ dans les pronoms personnels comme dans illils [i] et elle/elles [ $\varepsilon$ ];

- L'ouverture et le relâchement des voyelles /i, y, u/ comme dans rite [RIt], rut [RYt] et route $[\mathrm{Rut}])$;

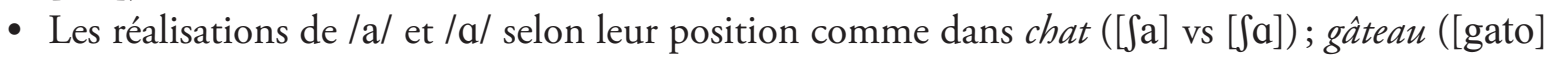
vs [ga:to]) et patte [pat] vs pâte [pa:t];

- Le dévoisement (et l'effacement) des voyelles /i, y, u/ comme dans université [yniversite] vs [ynivers(i)te];

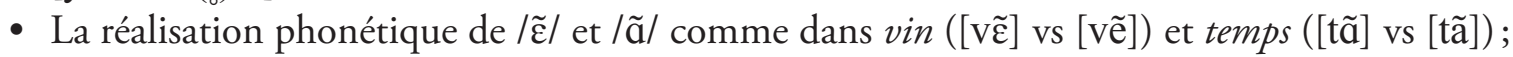

- La réalisation de mots en -oi et -ois comme dans le bois ([ləbwa] vs [ləbwa]).

Bigot et Papen (2013: 127) ajoutent que "aucun des autres traits typiques de la prononciation "populaire" du Québec, utilisés surtout dans les registres familiers, ne fait partie de la "norme” ». Finalement, ils concluent: "À la lumière de ces faits, il apparaît donc évident qu'une norme de prononciation du français québécois [...] n’est pas encore entièrement déterminée.» (Bigot et Papen 2013: 128)

\subsection{La grammaire}

En ce qui concerne la grammaire, il n'y a, à notre connaissance, qu'une étude sociolinguistique descriptive réalisée au sujet de cet aspect de la norme du français québécois oral: la thèse de Bigot (2008), dont les résultats ont été repris dans Bigot (2010) et Bigot (2011).

Rappelons tout d'abord que l'objectif de cette étude est de rendre compte des usages grammaticaux réels (c'est-à-dire des usages tirés d'un corpus oral et non inventés) de l'élite sociale et culturelle québécoise en situation de communication formelle, afin de vérifier si oui ou non, il existe une norme grammaticale orale, en français québécois. Pour cela, Bigot (2008) a réalisé une étude statistique basée sur l'examen d'un corpus de 110 entrevues journalistiques diffusées dans l'émission Le Point, sur le canal télévisuel de Radio-Canada ${ }^{15}$. Les variables observées par Bigot (2008) sont les suivantes (la première variante est vernaculaire, la seconde est standard ${ }^{16}$ ):

- La neutralisation de tous/tout en [tut], p. ex. : Ils sont tous [tut] vs [tus] venus;

- La neutralisation des démonstratifs ce, cet, cette en [stə], p. ex.: Cette [stə] vs [set] voiture là;

- La neutralisation du participe passé de faire en [fet], p. ex. : Il a fait [fet] vs [fe] son travail;

- L'alternance j'vais vs j'vas vs m'as (+ verbe à l'infinitif), p. ex. : J'vais vs j'vas vs m'as travailler;

15. Voir Bigot (2008: 167-174) pour plus de détails sur le corpus Le Point.

16. Voir Bigot (2008: 175-176) sur les variables qui ont été exclues de l'analyse, faute d'occurrences. 
- L'insertion du [l] non étymologique, p. ex. : Ça [l] vs Ø a pris du temps;

- La double négation, p. ex.: Il y a pas vs Øpersonnelrien;

- La locution conjonctive quand suivi de que, p. ex. : Quand que vs $\varnothing$ je rentre chez moi;

- Les relatives indirectes en que, p. ex. : C'est la personne que vs dont je te parlais;

- L'emploi du futur périphrastique dans les phrases positives, p. ex.: Je vais le voir vs je le verrai demain;

- L'emploi de l'auxiliaire avoir avec les verbes de mouvement, p. ex. : Elle a vs est passé(e) par Montréal;

- L'emploi du conditionnel après si, p. ex. : Si jaurais vs j'avais fait mon travail;

- Le présentatif en c'est des, p. ex. : C'est des vs ce sont des personnes très sympathiques;

- Les complétives et interrogatives indirectes, p. ex. : Je sais pas que c'est quelqu'est-ce que vs ce que je vais faire ou Je me demande que c'est quelqu'est-ce que vs ce que je vais faire;

- Les interrogatives (totales et partielles), p. ex. : Tu penses-tu?/ Tu penses? vs Penses-tu?/Est-ce que tu penses? et Que c'est que tu en penses?/Que c'est que c'est que tu en penses?/Qu'est-ce tu en penses? vs Qu'en penses-tu?/Qu'est-ce que tu en penses?

Pour chaque variable, Bigot (2008) a calculé le nombre de variantes standard - les variantes standard étant celles directement prescrites dans le Bon usage de Grevisse-Goose - et celui des variantes vernaculaires. Il dégage les pourcentages présentés dans le tableau ci-dessous.

Tableau 2: Pourcentages de variantes standard pour chaque variable (tiré de Bigot 2008: 311)

\begin{tabular}{|l|c|c|}
\hline \multicolumn{1}{|c|}{ Variable linguistique } & Taux moyens en \% & n/N \\
\hline Réalisation des démonstratifs ce et cettel cet en [sə] et [set] & 98,5 & $520 / 528$ \\
\hline Réalisation de tous en [tus]/[tu] et de tout en [tu] & 97,1 & $437 / 450$ \\
\hline Emploi de la conjonction quand... sans que & 96,5 & $249 / 258$ \\
\hline Emploi des relatives et interrogatives indirectes de référence & 95,4 & $415 / 435$ \\
\hline Emploi de l'auxiliaire être devant les verbes de mouvement & 94,5 & $121 / 128$ \\
\hline Emploi de l'imparfait après si au conditionnel & 94,3 & $66 / 70$ \\
\hline Absence du/l/non étymologique devant ça & 92,0 & $127 / 138$ \\
\hline Emploi de la simple négation devant rien/personne & 91,9 & $57 / 62$ \\
\hline Emploi de dont dans les relatives indirectes & 90,5 & $47 / 52$ \\
\hline Réalisation du participe passé fait en [fe] & 89,9 & $213 / 237$ \\
\hline Réalisation de je vais en j’vais/je vais & 79,7 & $67 / 84$ \\
\hline Emploi des interrogatives directes de référence & 79,6 & $191 / 240$ \\
\hline Emploi de ce sont des... au présentatif & 39,6 & $57 / 144$ \\
\hline Emploi du futur synthétique dans les phrases positives & 21,1 & $151 / 715$ \\
\hline
\end{tabular}

Les résultats du tableau 2 permettent de souligner que les locuteurs du corpus Le Point ont très largement favorisé les variantes de référence, et ce pour douze des quatorze variables grammaticales. Bigot (2008: 311-312) conclut alors que « [à] l'exception de la neutralisation du présentatif ce sont... en c'est... (39,6\%) et de l'utilisation du futur synthétique dans les phrases positives $(21,1 \%)$, la grammaire privilégiée par les locuteurs de notre corpus est celle décrite dans le Bon usage de Grevisse-Goose». 
Soulignons un dernier aspect de l'analyse de Bigot (2008). Les données statistiques qu'il a recueillies ont fait l'objet d'une analyse externe en fonction des trois facteurs extralinguistiques suivants: le sexe des locuteurs, leur âge et leur catégorie d'emploi ${ }^{17}$. Les résultats obtenus font ressortir que: 1) les locutrices du corpus Le Point tendent à utiliser davantage les variantes standard que leurs homologues masculins, 2) plus les locuteurs sont âgés, plus ils emploient les variantes standard, et 3) les interviewés utilisent, à l'oral, le même modèle grammatical normatif, et ce, quelle que soit la catégorie d'emploi à laquelle ils appartiennent (Bigot 2008: 313-316).

\section{Discussion}

Au regard des diverses propositions et définitions vues dans la première section, il parait difficile de trouver un terrain d'entente entre les partisans d'une norme internationale et ceux qui favorisent une norme québécoise. Les descriptions réalisées jusqu’à présent à partir de corpus sociolinguistiques nous fournissent cependant plusieurs informations susceptibles de mener à une solution. Nous nous interrogeons donc maintenant sur les pistes à suivre si l'on veut résoudre le problème de la norme du français québécois oral ${ }^{18}$.

Tout d'abord, nous pensons qu'il convient de retenir que la question de la norme linguistique, au sens du bon usage, restera sans doute toujours un sujet de débat et de controverse. Le concept de norme étant avant tout un concept social (Pillon 2003), la norme telle qu'elle est prescrite dans les ouvrages de référence n'est qu'une représentation stratifiée des pratiques linguistiques (réelles ou fantasmées) ${ }^{19}$ d'une communauté qui la définit et qui l'adopte ou non. En cela, elle est logiquement portée à évoluer au gré des générations, ce qui implique qu'elle ne peut être fixée une bonne fois pour toutes ${ }^{20}$.

Toutefois, cela ne veut pas dire qu'il ne faille jamais la définir et la décrire. Bien au contraire, car sans définition ni modèle, il paraît très difficile d'instaurer une quelconque norme. Nous pensons que si la question de la norme orale du français québécois n'a pas encore permis d'arriver à un consensus, c'est en raison de deux problèmes majeurs. Le premier est que très rapidement, on a opposé deux normes, l'une internationale (proposée par l'OLF) et l'autre québécoise (proposée par l'AQPF), comme si ces dernières ne partageaient finalement aucun trait linguistique commun ${ }^{21}$. Or, comme l'indique Maleville (1982: 70), «établir des normes, c'est comparer». Si la polarisation entre le modèle international et le modèle québécois a persisté pendant de nombreuses années dans l'imaginaire collectif des langagiers, des intellectuels et d'une partie de la population (et nous sommes convaincu qu'elle persiste

17. Voir Bigot (2011:6) pour plus d'informations sur chaque catégorie.

18. Par corpus sociolinguistique, nous référons à une base de données linguistiques représentative des pratiques réelles de la population ciblée.

19. Voir notamment Moreau (1997) au sujet des normes fantasmées.

20. Par communauté, nous entendons «un système constitué d'agents et d'objets utilisés par les agents, structurés dans leurs représentations, reconnus par eux, et dans certains cas, aménagés par eux» (Baggioni et al. 1997: 88).

21. La position de l'OLF (1965) est d'ailleurs particulièrement radicale puisque les normalisateurs de l'époque excluent de la norme québécoise des traits de prononciation (notamment l'assibilation de / $/$ / et $/ \mathrm{d} /$ ) qui font directement partie du système phonologique du français québécois et qui ne font en rien l'objet de représentations sociales négatives au Québec. L'OLF (1965: 8) va jusqu’à écrire que ce sont des traits «de prononciations vicieuses qui exigent un travail de redressement articulatoire». L'organisme précise que «[c]'est dans le seul domaine du lexique qu'il est possible d'accepter des divergences, à la condition que les termes ainsi introduits soient construits selon la logique de la langue française» (OLF 1965: 9). Pour autant, le document de l'OLF (1965) ne fait que douze pages et ne balise concrètement que très peu la norme du français québécois. 
encore de nos jours), c'est parce qu'il aura fallu pas moins de 30 ans pour voir apparaître les premières études descriptives basées sur des corpus sociolinguistiques qui permettent de décrire les usages réels de locuteurs considérés comme potentiellement détenteurs de la norme du français québécois oral. Comme nous l'avons déjà souligné, ni l'OLF, ni l'AQPF n'ont proposé de description linguistique venant appuyer leur proposition. Il était donc, jusqu’à tout récemment, impossible de savoir quelles étaient les caractéristiques réelles de la norme du français québécois oral.

Le second problème vient du fait qu'on ne sait pas encore qui sont les locuteurs pleinement détenteurs de la norme du français québécois oral. Nous avons vu dans la première section que plusieurs propositions avaient été faites, mais qu'elles soulevaient plus de questions qu'elles n'en résolvaient. Pour notre part, nous réitérons notre position, déjà exposée dans Bigot (2008, 2010, 2011), en proposant que le français parlé par l'élite sociale et culturelle du Québec en situation de communication formelle (au sens d'Auger 1997: 153) soit le modèle qui se rapproche le plus de ce qui peut être défini comme norme du français québécois oral. Par élite sociale et culturelle du Québec, nous entendons des personnes dont la position sociale est élevée (mais dont le niveau de scolarité peut ne pas l'être) et dont la profession relève du domaine public, ce qui leur assure une cote maximale sur le marché linguistique 22 (par exemple: les avocats, les responsables d'entreprises, les professeurs, les sportifs de haut niveau, etc.) ${ }^{23}$. Deux réalités s'inscrivent dans notre perspective: une réalité sociale et culturelle québécoise (Petiot et Marchello-Nizia 1972: 112) dans laquelle la norme est définie à partir des usages (au sens de Landry 1972: 30) de locuteurs québécois, ainsi qu'une réalité sociolinguistiquement plus théorique (elle tient compte du registre de langue soutenu de locuteurs qui font partie des élites sociale et culturelle québécoises et qui possèdent donc le capital linguistique) mais non négligeable, dans laquelle «toute la structure sociale est présente dans les interactions (et par là dans le discours)" (Bourdieu 1982: 61)24.

D'un point de vue descriptif, nous pensons que les études sur la prononciation de Cox (1998), Reinke (2005) et Bigot et Papen (2013), ainsi que Bigot (2008) sur la grammaire orale, fournissent des points de repère importants dans un processus de description concrète de la norme du français québécois oral. Rappelons que Bigot et Papen (2013: 125) ont déterminé huit traits de prononciation typiques du parler des annonceurs de nouvelles de Radio-Canada, mais qu'il existe toujours six traits flottants. Il reste donc encore à statuer sur quelques traits susceptibles d'être tolérés ou non, mais on peut tout de même affirmer avoir un bon aperçu de la prononciation normative du français québécois et qu'une grosse partie du travail a été réalisée. Pour ce qui est de la grammaire, la thèse de Bigot (2008) démontre que les élites québécoises tendent à suivre le modèle prescrit dans les ouvrages tels que le Bon usage de Grevisse. Autrement dit, il n’y a aucun besoin de décrire cette partie de la norme québécoise, puisqu'elle semble identique à celle du reste de la francophonie.

Un problème de corpus se pose cependant. Les études qui ont traité de la prononciation ont pris en compte des locuteurs spécialisés et non des membres de l'élite québécoise, comme dans l'étude de Bigot (2008). Pour le moment, on ne sait pas s'il existe des différences entre la prononciation des lecteurs de nouvelles et celle des élites québécoises en situation de communication formelle, et il n'est pas impossible que certains points de divergence existent. Pour s'en assurer, il faudrait réaliser une nouvelle étude de corpus portant exclusivement sur la prononciation de ces élites québécoises. Nous sommes

22. Voir Bourdieu (1982) sur la notion de marché linguistique.

23. Les résultats de Bigot (2008: 316) démontrent notamment que les locuteurs du corpus «Le Point» ne se distinguent pas de façon significative en fonction de leur catégorie d'emploi.

24. Notons que notre définition se base également sur un échantillon plus large de la population québécoise que celui des lecteurs de nouvelles, tout en étant plus ciblé que «la majorité des Québécois», comme le proposait l'AQPF (1977). 
convaincu que les élites québécoises, lorsqu'elles sont en situation de communication formelle, alignent leur prononciation sur le même modèle que celui des annonceurs de nouvelles québécois de la SRC. Cela reste néanmoins à prouver, car si tel n'était pas le cas, il faudrait impérativement reposer la question des locuteurs représentatifs de la norme du français québécois.

\section{Conclusion}

Selon nous, il existe donc bel et bien une norme du français québécois oral. Elle se traduit par l'usage de certains traits typiques de la prononciation québécoise, mais, du point de vue de la grammaire, elle ne se différencie pas de la norme décrite dans les grands ouvrages prescriptifs. Nous rejoignons ainsi la position de l'OLF (1965) pour ce qui est de la grammaire, ainsi que celle de l'AQPF (1977), pour ce qui est de la prononciation. Par ailleurs, nous pensons que cette norme n'est pas uniquement employée par des locuteurs spécialisés, et qu'une partie plus large de la population québécoise l'utilise en contexte formel, ce qui la rend plus représentative et donc potentiellement plus accessible à toute la population.

Le problème qui se pose maintenant est certainement de faire adopter ce modèle par les agents qui véhiculent la norme, et notamment les enseignants ${ }^{25}$. Pour cela, nous croyons que l'Office québécois de la langue française (OQLF - anciennement OLF) devrait se prononcer sur la question de la norme du français québécois, et ce, en tenant compte des dernières recherches publiées. Nous pensons que cela fait partie de son mandat. Comme nous l'avons souligné ci-haut, il ne semble rester qu'à statuer sur quelques points de prononciation. Il serait donc tout à fait possible de concevoir, dans un avenir rapproché, un document dans lequel l'OQLF décrirait concrètement cette norme du français québécois oral, en précisant quels traits de prononciation font partie du bon usage québécois et quels traits n'en font pas partie. Pour la grammaire orale, une description détaillée est inutile et l'OQLF n'aurait qu'à lister les grands ouvrages qu'il considèrerait comme normatifs ${ }^{26}$. Enfin, il serait également pertinent d'ajouter des informations lexicales permettant de préciser le statut de certains mots couramment employés au Québec, mais parfois jugés fautifs (notamment "assir», "performer», "la motion" (dans le sens de «le geste»), etc.).

La rédaction de ce qui pourrait prendre la forme d'un guide pédagogique de la norme du français québécois oral aurait divers impacts. Tout d'abord, un tel ouvrage permettrait de briser un mythe qui laisse très souvent, peut-être même trop souvent, entendre que le parler des Québécois se résume à sa variété vernaculaire et que c'est cette variété vernaculaire qui fait office de norme. Ce document permettrait ainsi de renforcer l'enseignement du français langue maternelle, et plus particulièrement de sa variation stylistique, en indiquant ce qui accepté ou non dans la norme québécoise. Ceci n'est pas négligeable, car nous sommes d'avis qu'une part trop peu importante de la population québécoise possède une conscience accrue des enjeux liés à la variation stylistique, et qu'un trop grand nombre de Québécoises et de Québécois ne maîtrisent que partiellement les niveaux de langue du français québécois.

25. Sans remettre totalement en cause la formation des maîtres au Québec, nous devons admettre que nous avons très souvent rencontré des enseignants, tous niveaux confondus, qui employaient des structures grammaticales ainsi que des traits de prononciation typiquement vernaculaires en situation de communication formelle. Inversement, reprendre des étudiants utilisant des variantes québécoises vernaculaires en situation informelle de communication constituerait aussi une erreur de pratique pédagogique.

26. Cette liste d'ouvrages recommandés devrait être cependant complétée par des informations permettant de statuer sur l'usage de tournures particulièrement fréquentes en français québécois (telles que Avoir su, je ne serais pas venu), afin de guider le mieux possible les agents véhiculant la norme. 
Un tel guide fournirait également des balises très intéressantes à suivre pour l'enseignement du français langue seconde en contexte québécois. En effet, les cours de français langue seconde sont, selon ce que nous avons pu observer dans les universités montréalaises, encore très généralement basés sur le français de France, et le matériel pédagogique localisé (c'est-à-dire adapté à la variété pratiquée par la population locale) pour le Québec se fait rare ${ }^{27}$. Établir clairement une norme de prononciation du français québécois permettrait, par exemple, de concevoir des cours de français langue seconde dans lesquels on enseignerait certains traits spécifiques de la norme québécoise (comme l'assibilation de /t/ et $/ \mathrm{d} /$ ), ce qui favoriserait directement l'intégration linguistique des apprenants. La création de tels cours nécessiterait toutefois, nous en convenons, un débat de la part des didacticiens spécialistes de l'enseignement du français langue seconde.

Enfin, nous restons convaincu que la publication d'un nouveau guide descriptif complet sur la norme du français québécois oral (et même plus généralement sur la norme du français québécois) permettrait sans aucun doute de diminuer l'insécurité linguistique des Québécois, insécurité linguistique qui, bien qu'elle tende à s'estomper depuis quelques années (Maurais 2008: 13), reste, selon nous, très présente. L’enjeu n'est pas uniquement linguistique, car nous pensons qu'officialiser une norme locale, c'est non seulement s'affranchir d'un modèle qui ne nous représente pas, mais c'est aussi reconnaître et faire reconnaître davantage les spécificités sociales et culturelles de la population qui pratique cette norme.

\section{Références bibliographiques}

Association québécoise des professeurs de français. 1977. «Le congrès du dixième anniversaire. Les résolutions de l'assemblée générale.». Québec français 28: 10-12. Consulté le 27 octobre 2016 de https:/id.erudit. org/iderudit/56625ac

Auger, J. 1997. "Formel vs informel». Dans Sociolinguistique. Concepts de base, sous la direction de M.-L. Moreau. Liège: Mardaga: 152-153.

Auger, P. 1988. «Identification linguistique des Québécois et dictionnaire général d'usage ou le comment du sentiment linguistique des Québécois en 1986». Revue québécoise de linguistique théorique et appliquée 7 (1): 55-69.

Baggioni, D., M.-L. Moreau et D. de Robillard. 1997. «Communauté linguistique». Dans Sociolinguistique. Concepts de base, sous la direction de M.-L. Moreau. Liège: Mardaga: 88-93.

Barbaud, P. 1998. «Dissidence du français québécois et évolution dialectale». Revue québécoise de linguistique 26 (2): Représentation de la langue et légitimité linguistique: le français et ses variétés nationales, dirigé par C. Verreault et L. Mercier: 107-128. doi : http://dx.doi.org/10.7202/603156ar

Bertrand, G. 2005. «La radio et la télévision: modèles linguistiques ou miroirs de société?». Dans Le français au Québec. Les nouveaux défis, sous la direction de A. Stefanescu et P. Georgeault. Québec: Fides: 445-460.

Bigot, D. 2008. "Le Point" sur la norme grammaticale du français québécois oral. Thèse de doctorat, Université du Québec à Montréal.

Bigot, D. 2010. "La norme grammaticale du français québécois oral: des questions, une réponse». Dans Vues sur les français d'ici, sous la direction de C. Leblanc, F. Martineau et Y. Frenette. Québec: Presses de l'Université Laval: 9-30.

27. À notre connaissance, seules quatre méthodes localisées existent actuellement sur le marché: Interaction (St Onge et al. 2011), Écho A1 pour l'Amérique du Nord (Girardet et al. 2014), Écho A2 pour l'Amérique du Nord (Girardet et al. 2015) et Par ici (Desjardins et al. 2015). Notons que trois de ces quatre méthodes n’existent que depuis 2014. 
Bigot, D. 2011. «De la norme grammaticale du français parlé au Québec». Arborescences 1 : Identités linguistiques, langues identitaires: à la croisée du prescriptivisme et du patriotisme, sous la direction de A.-M. Brousseau. doi: http://dx.doi.org/10.7202/1001939ar

Bigot, D. et R. A. Papen. 2013. "Sur la "norme” du français oral au Québec (et au Canada en général) ». Langage et société 4 (146): 115-132. doi : http://dx.doi.org/10.3917/ls.146.0115

Bouchard, P. et J. Maurais. 1999. «La norme et l'école. L'opinion des Québécois.». Terminogramme (91-92): La norme du français au Québec. Perspectives pédagogiques, sous la direction de C. Ouellon: 91-116.

Bourdieu, P. 1982. Ce que parler veut dire. L'économie des échanges linguistiques. Paris: Fayard.

Cajolet-Laganière, H. et P. Martel. 1995. La qualité de la langue au Québec. Québec: IQRC.

Comité interministériel sur la situation de la langue française. 1996. Le français langue commune. Enjeu de la société québécoise. Québec: Direction des communications du ministère de la Culture et des Communications.

Conseil de la langue française. 1990. L’aménagement de la langue: pour une description du français québécois. Québec: Conseil de la langue française.

Conseil supérieur de la langue française. 2007. Bulletin 23 (1). Juin.

Corbeil, J.-C. 1983. «Éléments d'une théorie de la régulation linguistique». Dans La norme linguistique, sous la direction de É. Bédard et J. Maurais. Québec: Conseil de la langue française: 281-304.

Corbeil, J.-C. 1986. "Le régionalisme lexical: un cas privilégié de variation linguistique». Dans Lexicographie québécoise. Bilan et perspectives, sous la direction de L. Boisvert, C. Poirier et C. Verreault. Québec: Presses de l'Université Laval: 55-61.

Corbeil, J.-C. 2007. L'embarras des langues. Origine, conception et évolution de la politique linguistique québécoise. Montréal: Québec Amérique.

Cox, T. B. 1998. "Vers une norme pour un cours de phonétique française au Canada». Revue canadienne des langues vivantes 54 (2): 175-197. doi: http://dx.doi.org/10.3138/cmlr.54.2.172

Desjardins, N., R. Sauvé et M. Usereau. 2015. Par ici. La méthode de français pour la réalité nord-américaine. Montréal: Les éditions MD.

Dor, G. 1998. Les qui qui et les que que ou le français torturé à la télé. Un troisième et dernier essai sur le langage parlé des Québécois. Montréal: Lanctôt.

Dubuc, R. 1990. "Le Comité linguistique de Radio-Canada». Dans Dix études portant sur l'aménagement de la langue au Québec. Québec: Conseil de la langue au Québec: 131-155.

Dumas, D. 2006. "La transcription phonétique du dictionnaire Franqus». Revue canadienne de linguistique appliquée 9 (2)/Revue de l'Université de Moncton 37 (2): Les variétés de français en Amérique du Nord. Evolution, innovation et description, sous la direction de R. A. Papen et G. Chevalier: 99-110. doi: http:// dx.doi.org/10.7202/015841ar

Forget, D. 1979. "Quel est le français standard au Québec?». Dans Le français parlé: études sociolinguistiques, sous la direction de P. Thibault. Edmonton: Linguistic Research Inc: 153-161.

Gendron J.-D. 1990. "Modèles linguistiques, évolution sociale et normalisation du langage», Dans Langue et identité: les français et les francophones d'Amérique du Nord, sous la direction de N. Corbett. Québec: Presses de l'Université Laval: 369-388.

Gendron J.-D. 2014. La modernisation de l'accent québécois: de l'accent traditionnel au nouvel accent: 1841-1960. Québec: Presses de l'Université Laval.

Girardet, J., C. Gibbet, S. Callet, F. Olivry, D. Liakin et N. Liakina. 2014. Écho A1 pour l'Amérique du Nord. Paris: Clé International.

Girardet, J., C. Gibbet, S. Callet, F. Olivry, D. Liakin, N. Liakina et H. Boivin. 2015. Écho A2 pour l'Amérique du Nord. Paris: Clé International.

Labov, W. 1976. Sociolinguistique. Paris: Minuit.

Lamonde, D. 2004. Anatomie d'un joual de parade. Le bon français d'ici par l'exemple. Montréal: Varia.

Landry, L. 1972. Québec français ou Québec québécois?. Montréal: Les presses libres.

Léard, J.-M. 1995. Grammaire québécoise d'aujourd'hui. Comprendre les québécismes. Montréal: Guérin. 
Maleville, N. 1982. «Du barbarisme à la sociabilité ou ce qui joue un rôle dans l'établissement des normes». Le français dans le monde 169: 67-72.

Martel, G., K. Reinke, D. Deshaies, L. Ménard et C. Émond. 2010. «Variations sociodiscursives dans la mise en scène de l'information télévisée». Dans Hétérogénéité et homogénéité dans les pratiques langagières: mélanges offerts à Denis Deshaies, sous la direction de W. Remysen et D. Vincent. Québec: Presses de l'Université Laval: $87-114$.

Maurais, J. 1986. «Régionalismes et langue standard». Dans Lexicographie québécoise. Bilan et perspectives, sous la direction de L. Boisvert, C. Poirier et C. Verreault. Québec: Presses de l'Université Laval: 79-88.

Maurais, J. 1987. «L’expérience québécoise d'aménagement linguistique». Dans Politique et aménagement linguistique, sous la direction de J. Maurais. Québec et Paris: Conseil de la langue française et Le Robert:359-416.

Maurais, J. 2008. Les Québécois et la norme. L'évaluation par les Québécois de leurs usages linguistiques. Québec: Gouvernement du Québec.

Moreau, M.-L. 1997. «Les types de normes». Dans Sociolinguistique. Concepts de base, sous la direction de M.-L. Moreau. Liège: Mardaga: 218-223.

Nemni, M. 1998. "Le français au Québec: représentation et conséquences pédagogiques». Revue québécois de linguistique 26 (2): Représentation de la langue et légitimité linguistique: le français et ses variétés nationales, dirigé par C. Verreault et L. Mercier: 151-175. doi : http://dx.doi.org/10.7202/603158ar

Office de la langue française. 1965. Norme du français parlé et écrit au Québec. Cahiers de l'Office de la langue française 1. Québec: Gouvernement du Québec.

Petiot, G. et C. Marchello-Nizia. 1972. «La norme et les grammaires scolaires». Langue française (16) : 99-115. Consulté le 27 octobre 2016 de http://www.persee.fr/doc/lfr_0023-8368_1972_num_16_1_5707

Pillon, V. 2003. Normes et déviance. Paris: Bréal.

Reinke, K. 2005. La langue à la télévision québécoise: aspects sociophonétiques. Québec: Gouvernement du Québec.

Santerre, L. 1981. «Le français québécois: langue ou dialecte?». Québec français (41): 23-27. Consulté le 27 octobre 2016 de http://id.erudit.org/iderudit/57121ac

Santerre, L. 1990. «Le français québécois: langue ou dialecte?». Dans Langue et identité. Le français et les francophones d'Amérique du Nord, sous la direction de N. Corbett. Québec: Presses de l'Université Laval: 29-33.

Stefanescu, A. et P. Georgeault. 2005. Le français au Québec. Les nouveaux défis. Québec: Fides.

St. Onge, S., R. St. Onge, S. Kaménova et D. Kadri. 2011. Interaction. Révision de grammaire française: première édition canadienne. Toronto: Nelson Education.

Trudgill, P. 1995. Sociolinguistics. An Introduction to Language and Society, $3^{\mathrm{e}}$ édition. Harmondsworth: Penguin Books.

Warnant L. 1987. Dictionnaire de la prononciation française dans sa norme actuelle. Paris: Duculot.

Verreault, C. 1999. «L'enseignement du français en contexte québécois: de quelle langue est-il question?». Terminogramme (91-92): La norme du français au Québec. Perspectives pédagogiques, sous la direction de C. Ouellon: 21-40.

\section{Liste des abréviations}

AQPF: Association québécoise des professeurs de français

CLF: Conseil de la langue française

CSLF: Conseil supérieur de la langue française

OLF: Office de la langue française

OQLF: Office québécois de la langue française

RDI : Réseau de l'information

SRC: Société Radio-Canada

TQS: Télévision Quatre-Saisons

TVA: Téléviseurs-Associés 